\title{
Morning Serum Melatonin Values in Multiple Sclerosis: A Biochemical Evaluation
}

\author{
Yasemin Gulcan Kurt, Mehmet Agilli' ${ }^{1}$, Fevzi Nuri Aydin ${ }^{2}$
}

$\mathrm{W}$ e read with great interest the article by Farhadi et al. ${ }^{[1]}$ entitled, "Serum levels of melatonin and cytokines in multiple sclerosis." The authors have reported that serum melatonin levels in the morning are decreased in multiple sclerosis (MS), and they have not find significant correlation between the melatonin levels and other cytokines. In their paper, the authors hypothesize that the decrease of melatonin could be a factor in the inflammatory reactions in the pathologic process of MS. We would like to make some additions to the paper in respect to the interpretations of biochemical markers and the study design.

Melatonin is an antioxidant hormone predominantly produced by the pineal gland. In humans, melatonin secretion occurs in darkness. Melatonin secretion reaches a peak from 2 a.m. to 4 a.m. ${ }^{[2]}$ Synthesis and secretion of melatonin is inhibited in the morning, especially by light falling on the retina. ${ }^{[2]}$ Melatonin is rapidly cleared from blood by a single passage through the liver. After this passage, melatonin is converted to the sulfated metabolites. The 6-hydroxymelatonin sulfate (6-HMS) is the major hepatic metabolite of melatonin which is excreted mainly in urine. Because of these reasons, the morning serum samples are not useful for evaluating serum melatonin levels. But urine 6-HMS could be a useful marker for the evaluating the pineal gland activity for the desired period. ${ }^{[3,4]}$
Sleep disorders, depression, and loss of appetite are the most common symptoms of MS. ${ }^{[5]}$ Sleep disorders may alter melatonin secretion and circadian rhythm. Also, consuming foods rich in melatonin, such as banana, orange, and pineapple, may increase the serum melatonin levels significantly. ${ }^{[2]}$ Patients with sleep disorder and/or loss of appetite in the MS group should be excluded. Confounding factors should be reconsidered for melatonin measurements and should be mentioned in the paper.

In conclusion, giving explanation of these concerns will certainly provide clearer information for the readers.

\section{REFERENCES}

1. Farhadi N, Oryan S, Nabiuni M. Serum levels of melatonin and cytokines in multiple sclerosis. Biomed J 2014;37:90-2.

2. Pandi-Perumal SR, Sirinivasan V, Maestroni GJ, Cardinali DP, Poeggeler B, Hardeland R. Melatonin: Nature's most versatile biological signal? FEBS J 2006;273:2813-38.

3. Ozkan E, Yaman H, Cakir E, Deniz O, Oztosun M, Gumus S, et al. Plasma melatonin and urinary 6-hydroxymelatonin levels in patients with pulmonary tuberculosis. Inflammation 2012;35:1429-34.

4. Aydin I. Can the morning serum melatonin values reflect inflammatory activity: A biochemical evaluation? Rheumatol Int 2014;34:1033.

5. Veauthier C, Paul F. Sleep disorders in multiple sclerosis and their relationship to fatigue. Sleep Med 2014;15:5-14.

From the Department of Biochemistry, Gulhane Military Medical Academy School of Medicine, Ankara, Turkey; ${ }^{1}$ Department of Biochemistry, Agri Military Hospital, Agri, Turkey; ${ }^{2}$ Department of Biochemistry, Sirnak Military Hospital, Sirnak, Turkey Received: Jun. 21, 2014; Accepted: Nov. 06, 2014

Correspondence to: Asst. Prof. Yasemin Gulcan Kurt, Department of Biochemistry, Gulhane Military Medical Academy School of Medicine, Ankara, Turkey. GATA, Biyokimya Anabilim Dali, 06018, Etlik, Ankara, Turkey. Tel: 90-312-3043304; Fax: 90-312-3043700; E-mail: ygkurt@gata.edu.tr

DOI: $10.4103 / 2319-4170.145761$ 